\title{
Claves de la exportación de fuerza de trabajo calificada en el capitalismo contemporáneo: lecciones de la experiencia mexicana
}

\author{
RAÚL DELGADO WISE* \\ MÓNICA GUADALUPE CHÁVEZ ELORZA**
}

RESUMEN: Bajo la égida neoliberal se genera una profunda reestructuración de los sistemas de innovación, con epicentro en Estados Unidos, que agudiza y acentúa las relaciones de intercambio desigual en el horizonte Norte-Sur. La exportación de fuerza de trabajo y, en particular, la exportación de fuerza de trabajo altamente calificada proveniente de los países periféricos, configuran un nuevo peldaño en la división internacional del trabajo. El propósito de este trabajo es contribuir al análisis de esta problemática, develando los mecanismos a través de los cuales las grandes corporaciones multinacionales han logrado incrementar ostensiblemente sus ganancias, mediante la incorporación — directa o indirecta — de una masa creciente de científicos y tecnólogos de la periferia. Esta trama, que se ilustra a partir del caso mexicano, revela no sólo la emergencia de nuevos mecanismos para abaratar costos y transferir riesgos y responsabilidades, sino también la apertura de canales para avanzar hacia una creciente mercantilización y apropiación privada de bienes comunes intangibles.

PALABRAS CLAVE: reestructuración de los sistemas de innovación, bienes comunes intangibles, migración calificada mexicana, globalización neoliberal, intercambio desigual.

* Profesor de la Unidad Académica en Estudios del Desarrollo de la Universidad Autónoma de Zacatecas y coordinador de la Cátedra Unesco sobre Migración, Desarrollo y Derechos Humanos, México.

**Investigadora posdoctoral en la Unidad Académica en Estudios del Desarrollo de la Universidad Autónoma de Zacatecas, con apoyo del Conacyt, México. 
ABSTRACT: Under the neoliberal aegis, a profound restructuring of systems of innovation was carried out, from its epicentre in the United States, which intensified and accentuated unequal trade relationships along the North-South axis. The export of labor and, in particular, the export of highly-skilled labor originating in the peripheral countries, heralded a new phase in the international division of labor. The aim of this study is to contribute to the analysis of this problematic, revealing the mechanisms through which large multinational corporations have been able to significantly increase their profits through the incorporation — direct or indirect — of a growing body of scientists and technologists from the periphery. This scheme, which we illustrate using the case of Mexico, shows not only the emergence of new mechanisms to reduce costs and transfer risks and responsibilities, but also the opening of channels, moving toward a growing commercialization and private appropriation of intangible public goods.

KEY WORDS: restructuring of innovative systems, intangible public goods, Mexican skilled migration, neoliberal globalization, unequal exchange. 


\section{INTRODUCCIÓN}

sistimos a una nueva época en la organización, orientación y apropiación del trabajo científico y tecnológico. Las dinámicas de internacionalización del capital propias de la globalización neoliberal han producido modificaciones sustanciales en la división internacional del trabajo que trastocan, en sus fibras más íntimas, las relaciones centro-periferia. En múltiples sentidos, la noción de sistema nacional de innovación ha sufrido profundas transformaciones que obligan a una reflexión crítica acerca de las maneras en que se generan los conocimientos científicos y tecnológicos en la actualidad y cómo son convertidos en fuentes de valorización por y para un grupo cada vez más reducido de grandes corporaciones multinacionales al amparo de los gobiernos imperiales, encabezados por Estados Unidos. Lo significativo de este complejo entramado, a cuyo desentrañamiento este artículo pretende contribuir, es que, paradójicamente, hay una creciente capacidad científica asentada o proveniente de los países periféricos o emergentes, el Sur global, la cual, lejos de contribuir al desarrollo de sus respectivos contextos nacionales, está dando lugar a nuevas modalidades de intercambio desigual que agudizan las asimetrías entre países y aumentan las desigualdades sociales. Este fenómeno, clave para la comprensión y transformación de la geopolítica de la producción del conocimiento, se relaciona con las dinámicas de mercantilización de la fuerza de trabajo científica y tecnológica que caracterizan al capitalismo contemporáneo y que imprimen un sello peculiar a la cuestión de la dependencia hoy. Pero también se vincula a una privatización sin precedentes de los bienes comunes intangibles o intelectuales (conocimientos, saberes, tradiciones y leguajes, entre otros), aspecto medular de la estrategia de dominación imperialista que caracteriza al capitalismo contemporáneo (Ramis, 2014; Foladori, 2014).

El análisis de la experiencia mexicana reviste particular relevancia en esta perspectiva. A raíz de la implantación de los programas de ajuste estructural en México, la migración mexicana a Estados Unidos experimenta un crecimiento sin precedentes. La implantación del Tratado de Libre Comercio 
de América del Norte (TLCAN) acentuó aún más este fenómeno al grado de convertir a México en el primer país de emigrantes del mundo. Esta desbordante dinámica expulsora trajo consigo profundos cambios cualitativos en el fenómeno migratorio asociados a procesos de transformación social de gran calado. Uno de ellos, que ha recibido relativamente poca atención en la literatura, es el referente a la migración altamente calificada, la cual exhibió en el curso de las últimas dos décadas un ritmo de crecimiento superior al de la migración mexicana en general.

El propósito de este trabajo es incursionar en el análisis de este fenómeno desde una perspectiva analítica comprehensiva y crítica. Nos interesa, ante todo, desentrañar el papel desempeñado por la fuerza de trabajo intelectual proveniente de México - y en general de los países periféricosen el proceso de reestructuración de los sistemas de innovación que se despliega en el marco de la globalización neoliberal y bajo la batuta de las grandes corporaciones multinacionales. Consideramos que bajo este mirador analítico es posible profundizar en la naturaleza y características de la migración altamente calificada (y en un sentido más general, de la exportación de fuerza de trabajo intelectual), en contraste con la perspectiva dominante que descontextualiza su análisis y lo restringe a un supuesto tránsito de la pérdida de cerebros a la circulación de talentos y a una eventual ganancia de cerebros a favor de los países emisores.

La hipótesis central de nuestro argumento es que la exportación de capacidad científico-tecnológica configura una fase superior de la estrategia de reestructuración emprendida por las grandes corporaciones multinacionales tendiente a abaratar los costos laborales mediante el recurso a fuerza de trabajo barata proveniente de países y regiones periféricas o emergentes; situación que, lejos de favorecer el desarrollo de los países de origen, ha dado lugar a la emergencia de nuevas formas de intercambio desigual y de dependencia en el horizonte Sur-Norte. Este proceso se asocia, a su vez, a la apertura de una nueva fase regresiva en el desarrollo capitalista caracterizada por la apropiación y creciente privatización de los bienes comunes en general y de los bienes comunes intangibles en particular. En esta perspectiva, bajo la égida neoliberal asistimos al "[...] tránsito definitivo de la patente o del

$6 \frac{\text { SEGUNDO SEMESTRE } 2015}{\text { MIGRACIÓN Y DESARROLLO NÚM. } 25}$ 
derecho de autor como incentivo o protección del creador y su obra al monopolio rentista de un conglomerado corporativo» (Ramis, 2014: 117).

\section{EXPORTACIÓN DE FUERZA DE TRABAJO E INTEGRACIÓN México-Estados Unidos}

Para nuestros fines analíticos resulta fundamental traer a colación algunos rasgos esenciales del proceso de integración de la economía mexicana a la estadounidense impulsado a lo largo de las últimas tres décadas. Este proceso, signado por la implantación de las reformas neoliberales en el país y la suscripción y puesta en marcha del TLCAN, se caracteriza por un triple movimiento.

Primer movimiento:

desmantelamiento y reinserción de la economía mexicana

Un rasgo insoslayable del curso que sigue el neoliberalismo en México es el acelerado desmantelamiento del aparato productivo, comercial y de servicios del país orientado al mercado doméstico para dar paso a una drástica reorientación de la economía hacia el mercado internacional. Este vuelco de timón hizo posible que, en relativamente corto tiempo, la economía mexicana se convirtiera en la principal exportadora de América Latina y la decimosegunda del mundo. A primera vista, se trata no sólo de un viraje hacia un nuevo dinamismo exportador, sino de un avance sin precedentes hacia una plataforma de exportación de carácter secundario-exportador, integrada en un 80 por ciento por bienes manufacturados, de los cuales casi un 40 por ciento son clasificados como bienes difusores de progreso tecnológico (Delgado Wise y Márquez, 2007).

Los arquitectos o artífices del Consenso de Washington han exaltado el caso mexicano como un caso paradigmático de «éxito» en la implantación de las reformas neoliberales y un ejemplo de las bondades del «libre 
comercio». Sin embargo, esto refleja una visión distorsionada de la realidad, una ilusión óptica que nada tiene que ver con lo que verdaderamente representa el modelo exportador implantado en México. Para develar el auténtico carácter de las exportaciones mexicanas es necesario percatarse de que, en su inmensa mayoría, descansan sobre dos pilares:

- La industria maquiladora, conformada por plantas de ensamble asociadas a una estrategia de relocalización productiva, comercial y de servicios de las grandes corporaciones multinacionales, principalmente de origen estadounidense, con miras a aprovechar los bajos costos laborales prevalecientes en México. ${ }^{1}$ Este tipo de «industria» se distingue por un elevadísimo componente importado.

- La maquila encubierta referida a plantas manufactureras de exportación con procesos productivos relativamente más complejos que la maquila, como es el caso de los sectores automotor y electrónico, pero cuya operación se rige bajo un sistema de importaciones temporales similar al de la industria maquiladora.

Tanto la maquila como la maquila encubierta se distinguen por carecer de encadenamientos hacia delante y hacia atrás con el resto del aparato productivo, comercial y de servicios nacional, lo que les confiere el carácter de economías de enclave. Asimismo, una y otra están sometidas a dinámicas de precarización laboral y acusan elevados diferenciales salariales respecto a la manufactura estadounidense: 1/10 en el caso de la maquila y 1/7 en la maquila encubierta. El elevado componente importado de estas actividades, que representan entre 80 y 75 por ciento de las exportaciones manufactureras totales, hace que su contribución a la economía mexicana se reduzca esencialmente a una exigua derrama salarial, esto es, al valor de la fuerza de trabajo incorporado a las exportaciones (Cypher y Delgado Wise, 2011).

${ }^{1}$ Un pivote fundamental de la reestructuración de las grandes corporaciones multinacionales bajo la égida neoliberal es el desplazamiento de partes del proceso productivo, comercial y de servicios hacia países y regiones periféricas en función de los enormes diferenciales salariales imperantes a escala global, i.e., el llamado arbitraje laboral global (global labour arbitrage). Véase, por ejemplo, Foster, MacChesney y Jonna (2011) y Delgado Wise (2013).

$8 \frac{\text { SEGUNDO SEMESTRE } 2015}{\text { MIGRACIÓN Y DESARROLLO NÚM. } 25}$ 
De aquí que, tras el espejismo de una plataforma avanzada de exportaciones manufactureras, lo que verdaderamente exporta el país, a través de los productos de la maquila y la maquila encubierta, sea fuerza de trabajo sin que los trabajadores mexicanos salgan del país (Tello, 1986). Se trata, para decirlo en términos más precisos, de una modalidad de exportación indirecta de fuerza de trabajo.

Si a la exportación indirecta de fuerza de trabajo se le suma la exportación directa de fuerza de trabajo que se produce a través de la migración laboral, el verdadero contenido de las exportaciones mexicanas y la naturaleza de modelo exportador implantado en el país cobran su auténtico significado. En efecto, más que un modelo avanzado de exportación manufacturera, lo que en realidad se implanta en el país es un modelo exportador de fuerza de trabajo barata. Tal viraje no sólo entraña una regresión en la plataforma de exportación del país, sino que implica un ataque frontal a las condiciones de vida y de trabajo de la mayoría de los mexicanos (Delgado Wise y Cypher, 2007; Delgado Wise y Márquez, 2007; Cypher y Delgado Wise, 2011).

Segundo movimiento:

generación de un desbordante ejército laboral de reserva

Como ya anticipábamos, los saldos de la reestructuración neoliberal han sido nefastos para el grueso de la población mexicana: entre 2000 y 2010 se crearon en el país 2.1 millones de empleos formales, frente a una oferta potencial de 9.5 millones. Ello ha dado lugar a un desbordante crecimiento del ejército laboral de reserva, que a lo largo de este periodo se incrementó en 7.5 millones de trabajadores, de los cuales 1.5 millones son desempleados, 3.9 millones se ubican en las filas de la informalidad y 2.1 millones son migrantes internacionales. ${ }^{2}$ Esta situación, que de suyo plantea un escenario dramático para el país, se ha visto agravada por la crisis desencadenada en Estados Unidos a partir de 2008 y por las deportaciones masivas emprendidas

${ }^{2}$ Estimaciones basadas en el U.S. Bureau Census, Current Population Survey, March Supplement, 2000 y 2010, y Cepalstat, Estadísticas de Empleo para América Latina, 2000-2010. 
por la administración del presidente Obama (3.7 millones de deportados en el periodo 2009-2013, según cifras oficiales del U.S. Department of Homeland Security, 2014).

Más todavía: como corolario de la sobreoferta laboral consustancial a la dinámica de reestructuración neoliberal en México, de acuerdo con datos del Instituto Nacional de Estadística, Geografía e Informática (INEGI, 2015) en el tercer trimestre de 2014, el 58.1 por ciento de los trabajadores mexicanos se ubicaban en las filas de la informalidad, al tiempo que 63.6 por ciento de los trabajadores asalariados percibía ingresos menores a 1.8 dólares por hora, 79.4 por ciento laboraba jornadas semanales de 35 horas o mayores de 40 horas.

Cabe agregar que en el trasfondo de esta atroz embestida en contra de la clase trabajadora subyacen severos procesos de descomposición social, donde las fronteras con el crimen organizado se han vuelto cada vez más porosas.

Tercer movimiento:

desencadenamiento de la migración forzada

Bajo el modelo exportador de fuerza de trabajo barata, la migración de México a Estados Unidos ha exhibido un crecimiento exponencial. Este crecimiento se acentúa con la puesta en marcha del TLCAN (véase gráfica 1), situación que ha posicionado a México como el principal país de migrantes del mundo. Las dimensiones que alcanza el fenómeno migratorio resultan por lo demás elocuentes: en 2014 la población de origen mexicano que residía en Estados Unidos se estimaba en poco más 35 millones de personas, entre emigrantes -documentados o no- nacidos en México (11.4 millones) y ciudadanos estadounidenses de ascendencia mexicana (24 millones). Se trata de la diáspora más grande del mundo establecida en un país y, en este caso, el mayor país de inmigrantes del mundo.

La migración mexicana a Estados Unidos ha experimentado también cambios cualitativos de primera magnitud en cuanto a escolaridad, composición

$10 \frac{\text { SEGUNDO SEMESTRE } 2015}{\text { MIGRACIÓN Y DESARROLLO NÚM. } 25}$ 


\section{GRÁFICA 1}

Migración mexicana a Estados Unidos, 1840-2014

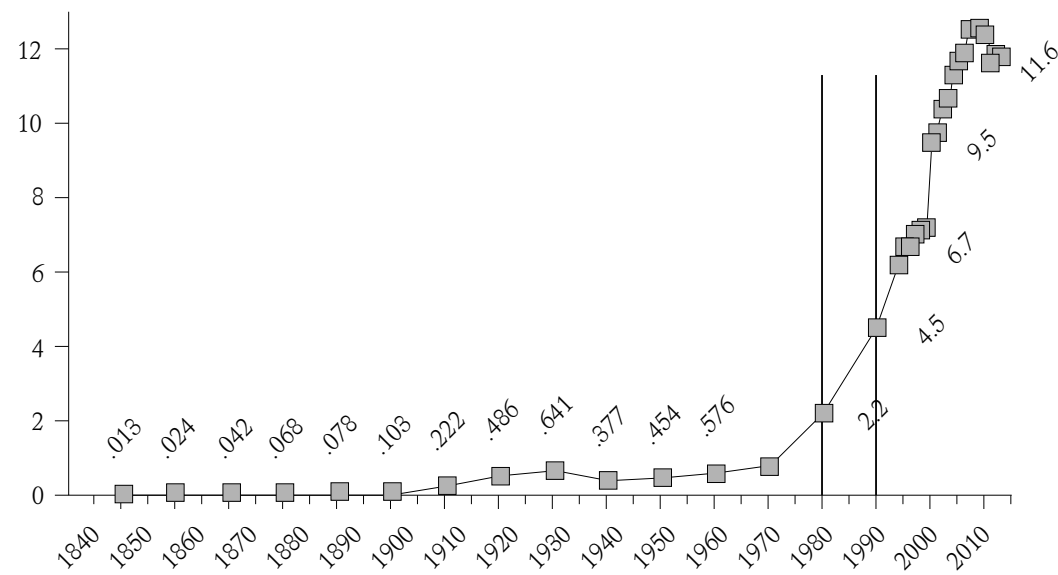

Fuente: Compilación de Censos Decenales, 1850-1990; Pew Hispanic Center, 1994-2010, Passel y Cohn, 2011; SIMDE, UAZ, de 2011 a 2014 con base en la CPS, suplemento de marzo.

étnica y de género, mayor permanencia de los flujos, etcétera. No obstante, su característica más importante es que esta migración asume, cada vez con mayor fuerza, el carácter de un desplazamiento forzado (Márquez y Delgado Wise, 2011). En este sentido, quienes participan en las corrientes migratorias son en su mayoría personas que han sido literalmente expulsadas de sus territorios, i.e., que abandonan sus lugares de origen por necesidad con la esperanza de acceder a medios de subsistencia u oportunidades de movilidad social allende las fronteras. Las condiciones en las que se producen estos desplazamientos conllevan múltiples riesgos y vicisitudes a lo largo del periplo migratorio - particularmente para los migrantes de baja calificación-, incluyendo su exposición permanente a condiciones de precarización laboral y exclusión social en los lugares de destino. Más aún, los migrantes internacionales están siendo crecientemente sometidos a políticas y prácticas de criminalización, racialización y discriminación, que no sólo los convierten en segmentos vulnerables y segregados, sino que muchas veces ponen en riesgo su propia vida. Tómese en consideración que más de la mitad de los migrantes mexicanos se ubican en la categoría de indocumentados 
(Passel y Cohn, 2011), lo que sitúa a México como el país con el mayor número de migrantes indocumentados del planeta, con todo lo que ello implica en términos de estigmatización y vulnerabilidad social. El retorno forzado derivado de las deportaciones masivas y la crisis en Estados Unidos agravan la situación descrita.

Es importante añadir que, en virtud de la prolongación hemisférica de la política de integración económica promovida por el gobierno imperial estadounidense, México se ha convertido en el principal corredor de tránsito migratorio del mundo. Se trata, en esencia, de un flujo de migración forzada sometido a condiciones aún mayores de vulnerabilidad, donde el creciente número de víctimas en territorio mexicano constituye un foco rojo, un gravísimo y vergonzante episodio en los anales de la historia del país, que no puede ni debe ser soslayado (Casillas, 2012; Castillo, 2005).

\section{REESTRUCTURACIÓN dE LOS SISTEMAS DE INNOVACIÓN BAJO LA ÉGIDA NEOLIBERAL}

El contexto en el que se despliega la migración calificada, particularmente aquella proveniente de los países periféricos o emergentes, está signado por una profunda reestructuración de los sistemas de innovación a escala global, con Estados Unidos a la cabeza y teniendo a las grandes corporaciones multinacionales como agentes centrales. Cuatro aspectos nodales caracterizan esta reestructuración:

1. Una mayor internacionalización y fragmentación de las actividades de investigación y desarrollo. En contraposición a los esquemas tradicionales de invención «a puertas cerradas» en departamentos de investigación y desarrollo enclavados en el seno de las grandes corporaciones, a esta tendencia se le conoce como innovación abierta en referencia a la apertura de funciones corporativas intensivas en conocimiento con la creciente participación de socios externos, tales como proveedores, clientes, subcontratistas, universidades, etcétera, para dar paso a la configuración de ecosistemas multisituados o redes de innovación (OECD, 2008). Bajo esta nueva plataforma se asume que

$12 \frac{\text { SEGUNDO SEMESTRE } 2015}{\text { MIGRACIÓN Y DESARROLLO NÚM. } 25}$ 
el conocimiento útil se halla ampliamente distribuido y que aun las organizaciones con mayor capacidad de desarrollo científico y tecnológico deben identificar y relacionarse con fuentes externas de conocimiento como parte medular de sus estrategias de innovación (Chesbrough, 2008: 1).

2. El establecimiento de ciudades científicas - como Silicon Valley en Estados Unidos y los nuevos Silicon Valley instaurados en regiones periféricas o emergentes, principalmente en Asia - donde se crean sinergias colectivas para acelerar las dinámicas de innovación (Sturgeon, 2003). Se trata, en el fondo, como lo destaca Anna Lee Saxenian (1996 y 2002), de un nuevo paradigma que se aparta de los viejos modelos "cerrados» de investigación y desarrollo incrustados en las grandes corporaciones y que abre el camino a una nueva cultura de la innovación basada en la flexibilidad, descentralización e incorporación, bajo diferentes modalidades, de nuevos y cada vez más numerosos jugadores que interactúan en espacios locales y transnacionales. En este último ámbito, las plataformas de innovación que se establecen en los países periféricos tienden a operar como extensiones de las plataformas instauradas en los países centrales, aprovechando ventajas salariales, tributarias y de otro tipo, lo que les confiere el carácter de maquiladoras científicas (Gallengher y Zarsky, 2007).

3. El desarrollo de nuevas formas de control de las agendas de investigación (mediante capital de riesgo, asociaciones y subcontrataciones, entre otros) y de apropiación de los productos del trabajo científico (vía adquisición de patentes) por las grandes corporaciones multinacionales a través del llamado strategic investment (Burgesmani, Christensen y Wheelwright, 2009). Resulta interesante observar al respecto que, aun cuando 76 por ciento de las patentes a universidades y alrededor de la mitad del total de patentes en años recientes en Estados Unidos fueron atribuidas a un inventor foráneo, 93 por ciento de los registros de las mismas fueron otorgados a corporaciones multinacionales (National Science Board, 2012; Partnership for a New American Economy, 2012; Rand Corporation, 2008; United States Patent and Trademark Office, 2012).

4. La expansión en el horizonte Sur-Norte de la fuerza de trabajo altamente calificada, en particular en las áreas de ciencia e ingeniería y el creciente reclutamiento - vía partnerships, outsourcing y offshoring - de esa fuerza de trabajo con especial significación en el caso de los países periféricos (Battelle, 2012). De hecho, la evolución de esta dinámica de reestructuración ha 
cristalizado en una nueva geografía de la innovación y en la investigación y el desarrollo científico-tecnológico asociados a ella. Desde 2011, Estados Unidos fue superado por China, Japón, Corea del Sur e India como principal inversor en investigación y desarrollo, y se espera que en 10 años más China supere a Estados Unidos en este renglón (Battelle, 2012). En lo referente a fuerza científico-tecnológica, de acuerdo con la American Community Survey en 2011, 1 de cada 3 maestros y 1 de cada 2 doctores en ciencias e ingeniería en Estados Unidos era extranjero - principalmente de países periféricos o emergentes-, al tiempo que fuera de Estados Unidos, destacadamente en China y la Unión Europea, se estaban preparando mayores contingentes de doctores en estas áreas del conocimiento que en el coloso de Norteamérica (Freeman, 2008; Galama y Hosek, 2008).

Es pertinente subrayar que esta dinámica de reestructuración ha posibilitado a las grandes corporaciones multinacionales poner a un creciente contingente de trabajadores científico-tecnológicos de los países periféricos o emergentes a su servicio, transferir riesgos y responsabilidades y capitalizar ostensibles beneficios mediante la concentración de patentes. Bajo este nuevo andamiaje se ha venido produciendo una mercantilización y monopolización sin precedentes del trabajo científico, con una visión de corto plazo y ajena a toda preocupación social, donde la emigración física e indirecta de personal altamente calificado de los países periféricos o emergentes desempeña un papel cada vez más significativo. En similar tenor, esto se asocia a las inescrupulosas dinámicas de apropiación de bienes comunes intangibles, es decir, bienes que por su naturaleza son «[...] ilimitados, infinitos, inagotables, no consumibles, no devastados, no contradictorios entre sí y basados en la abundancia» (Ramis, 2014: 114), cuya producción se basa en "comunidades que producen "artefactos comunes" disponibles bajo régimen común, como es el caso del software libre» (Ramis, 2014: 112).

Como parte de este engranaje, las universidades públicas y privadas de los países periféricos o emergentes, y específicamente de aquellos países que, como México, se han plegado a las directrices que emanan del paradigma neoliberal, cuentan con un sistema universitario que refleja nítidamente los 
intereses hegemónicos de los Estados imperiales y sus brazos corporativos. Esta situación — que ha sido ampliamente documentada en la literatura- se expresa en los procesos de trabajo académico impuestos a las instituciones de educación superior a través de métricas, incentivos y formas de evaluación que minan los resquicios de autonomía que aún prevalecían en las universidades públicas (Ibarra Colado y Porter, 2007; Ibarra Colado, 2005; Delgado Wise, 2003). Ello, a su vez, guarda correspondencia con los valores que tradicionalmente han permeado el medio académico en el contexto capitalista y particularmente bajo las pautas impuestas por el régimen neoliberal: selectividad/exclusión, individualismo y meritocracia utilitaria. En este tenor, a contra sensu de un pensamiento crítico asociado a procesos de transformación social, se procrea un conservadurismo estéril y sumiso, donde los procesos de innovación y las prácticas «científicas» de educación, producción y distribución de conocimiento permanecen enclaustradas en los marcos del intelecto social hegemónico, ajeno a cualquier tentativa de preservación y acrecentamiento de los bienes comunes intangibles. Y a esto se aúna un tejido productivo nacional desarticulado y subordinado a directrices e intereses externos — como se plantea en el apartado anterior-, donde el campo para la creatividad y la innovación propia han sido prácticamente anulados, cooptados o relegados. Como resultado, se prohíja un campo laboral para los trabajadores de la ciencia y la tecnología sumamente estrecho y precarizado, que más allá de las limitaciones formativas que emanan del propio sistema universitario, tiende a abrir anchas avenidas para el desperdicio de talentos $y$, con mayor empuje cada vez, la emigración de fuerza de trabajo altamente calificada.

\section{DIMENSIONES Y CARACTERÍSTICAS DE LA MIGRACIÓN MEXICANA ALTAMENTE CALIFICADA A Estados UnIDOS}

México figura como el primer país de América Latina y sexto a nivel mundial por su volumen de migrantes altamente calificados hacia países de la 
Organización para la Cooperación y el Desarrollo Económicos (OCDE) (Dumont, Spielvogel y Widmaier, 2010). El número de profesionistas mexicanos residentes en Estados Unidos con licenciatura, grado asociado a licenciatura, maestría y doctorado asciende a 1.1 millones, lo que sitúa a México en el segundo sitio de este tipo de inmigrantes en ese país (véase gráfica 2). A nivel de posgrado, el contingente de mexicanos - aunque relativamente bajo frente a China e India - fue el que más se duplicó en la última década, para alcanzar en 2011 un total de 129,027 con nivel de maestría y 12,026 con el grado de doctor. Este monto equivale a 17 por ciento de todos los posgraduados con los que cuenta el país (Tuirán y Ávila, 2013a). A ello, de acuerdo con los datos aportados por la American Community Survey, se agregan 2.5 millones profesionistas de origen mexicano nacidos en Estados Unidos, de los cuales 328,298 cuentan con maestría y 26,050 con doctorado. Si comparamos estos montos con la población altamente calificada que reside en México, la proporción de maestros y doctores de origen mexicano en Estados Unidos (inmigrantes y nacidos en ese país) resulta de enorme y sorprendente significación: 55.2 por ciento y 29.7 por ciento del total, respectivamente (véase gráfica 3). A estas cifras se agregan 60,000 mexicanos altamente calificados con el estatus de migrantes temporales (Rodríguez, 2009).

\section{GRÁFICA 2}

Inmigrantes altamente calificados en Estados Unidos por país de origen

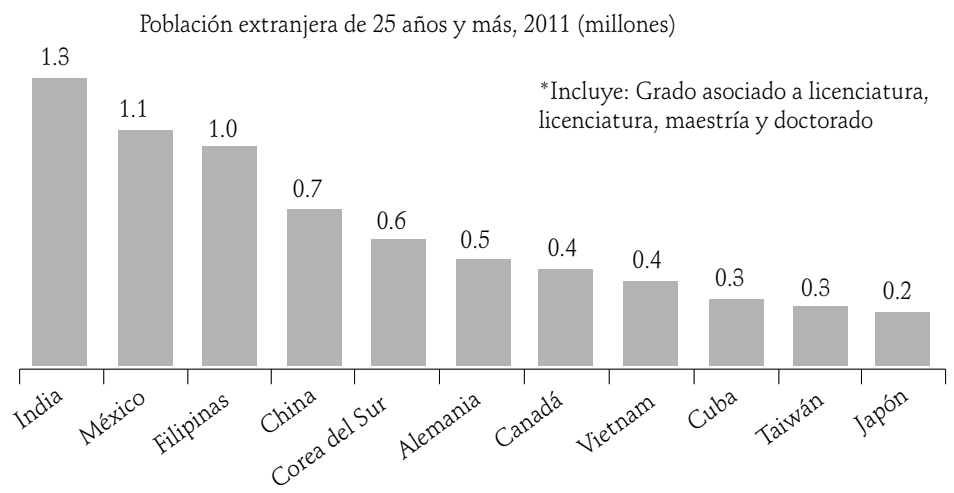

Fuente: SIMDE, UAZ. Estimación con base en U.S. Bureau of Census, American Community Survey, 2011.

$16 \frac{\text { SEGUNDO SEMESTRE } 2015}{\text { MIGRACIÓN Y DESARROLLO NÚM. } 25}$ 


\section{GRÁFICA 3}

Peso relativo de la población de origen mexicano con posgrado en Estados Unidos respecto a la radicada en México, 2010-2011

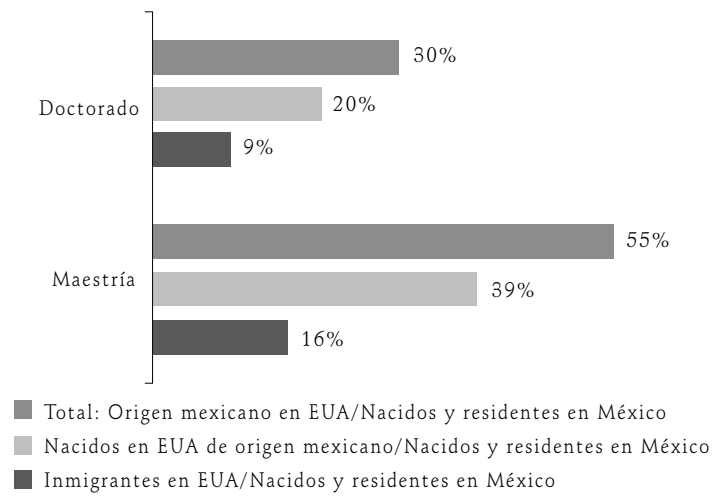

Fuente: SIMDE, UAZ. Estimación con base en INEGI, Censo de Población y Vivienda 2010 y U.S. Bureau of Census, American Community Survey, 2011.

Más allá de estas cantidades que de suyo son impactantes, es preciso destacar que apenas 54.7 por ciento de nuestros connacionales se ocupan en actividades profesionales o gerenciales en Estados Unidos (a nivel de posgrado la proporción se incrementa ostensiblemente: 74.3 por ciento) y que sus salarios, incluyendo los de los profesionistas de origen mexicano, tienden a ser más bajos que los del resto de los nativos e inmigrantes. A nivel de posgrado la situación salarial de los mexicanos y la población de origen mexicano en Estados Unidos sigue el mismo patrón. Con todo, cabe subrayar que al comparar estos salarios con los que reciben en nuestro país, la situación resulta aún más crítica (Estimación con base en U.S. Bureau of Census, American Community Survey, 2011).

A lo anterior se agrega la baja cobertura de educación superior que caracteriza al país (30 por ciento), no obstante el ostensible incremento que ha tenido el posgrado en la última década, lo que pone de relieve no sólo una limitada formación de fuerza de trabajo altamente calificada, sino una creciente pérdida, desperdicio y desaprovechamiento de talentos para el desarrollo nacional. Esta situación adquiere particular relevancia al considerar 
la proporción de posgraduados mexicanos en computación y análisis de sistemas, ciencias e ingeniería de origen mexicano en Estados Unidos (inmigrantes y nacidos en ese país) en comparación con los que residen en México, la cual representa 99, 66 y 47 por ciento, respectivamente (véase gráfica 4).

\section{GRÁFICA 4}

Peso relativo de la población de origen mexicano con posgrado en Ciencias e Ingeniería en Estados Unidos respecto a la radicada en México, 2010-2011

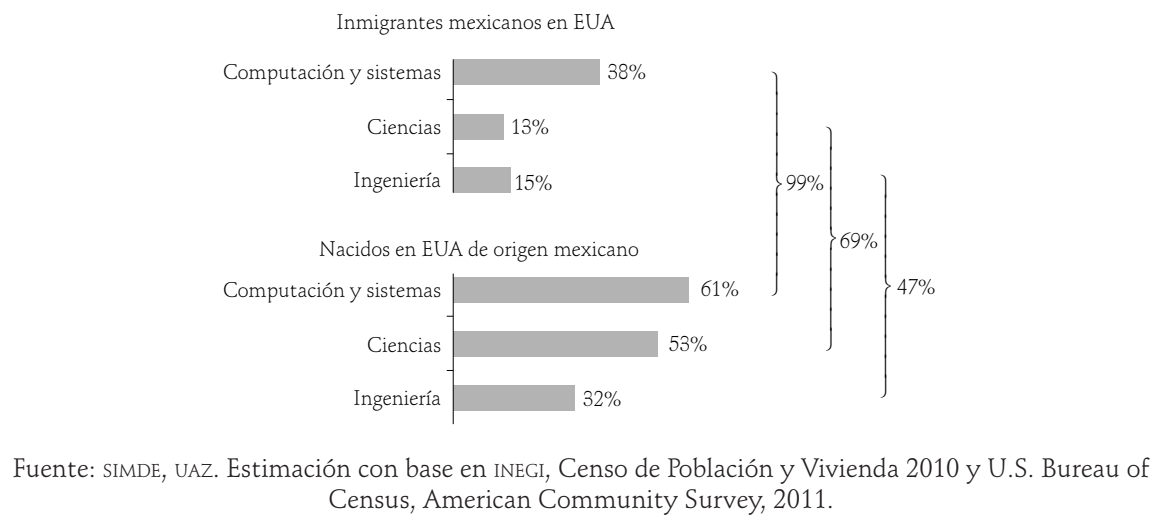

A diferencia de la migración mexicana en general, que se concentra casi en su totalidad en Estados Unidos (98 por ciento), la migración altamente calificada, no obstante que mayoritariamente se ubica en ese país, acusa una cierta diversificación geográfica hacia otros países de América, así como de Europa, Asia y Oceanía. De hecho, 19 por ciento de los posgraduados mexicanos que residen en el extranjero se ubicaban en 2013 fuera de Estados Unidos, de acuerdo con estimaciones basadas en censos provenientes del International Population Center de la Universidad de Minnesota y el Departamento de Asuntos Económicos y Sociales de la Organización de las Naciones Unidas (ONU). En respuesta a esta dispersión geográfica, el Instituto de los Mexicanos en el Exterior (IME) ha promovido la formación de la Red de Talentos Mexicanos en el Exterior, la cual cuenta con 27 capítulos y presencia en 12 países.

$18 \frac{\text { SEGUNDO SEMESTRE } 2015}{\text { MIGRACIÓN Y DESARROLLO NÚM. } 25}$ 


\section{Falacias de la circulación de cerebros}

En la discusión académica y política sobre migración calificada se ha venido abandonando el concepto de «fuga de cerebros» para reemplazarlo por la noción de «circulación de cerebros o de talentos» (Meyer, 2011; Saxenian, 2006). Mediante este viraje, el pesimismo y preocupación acerca de la emigración calificada Sur-Norte se ha transformado en un optimismo rampante que sustituye la noción de pérdida por la de ganancia. En este viraje subyace el supuesto de que el conocimiento es, en sí mismo, benéfico para todos y que el contacto con connacionales altamente calificados en el exterior genera sinergias que potencian el desarrollo del país de origen, sin importar dónde, cómo, en qué y para quién trabajen. Tanto el conocimiento como las agendas de investigación son concebidos como neutrales y, en similar tenor, la cuestión de la propiedad intelectual -i.e. la apropiación de los productos del trabajo científico/técnico- es menospreciada o simplemente ignorada. Más aún, la euforia desencadenada en torno a la «circulación de talentos» y el establecimiento de programas de acercamiento con las «diásporas calificadas» parten del supuesto de que la innovación crea, a través de procesos de incubación, sus propios encadenamientos con el sector productivo, comercial, financiero y de servicios.

Ninguno de los supuestos en los que se sustenta el optimismo de los promotores y epígonos de la «circulación de cerebros o de talentos» tiene asidero en la realidad del capitalismo contemporáneo, como claramente lo evidencian los siguientes datos y consideraciones referentes al caso mexicano:

- Después de China, México es el país con la mayor tasa de crecimiento de programas doctorales en el mundo: 17.1 por ciento entre 1998 y 2006 (Cyranoski et al., 2011).

- La oferta de programas de posgrado en el país asciende a 8,522, de los cuales 5,865 corresponden al nivel de maestría, 1,773 de especialidad y 884 de doctorado. Estos programas son ofertados por 1,423 instituciones, de las cuales 1,134 son particulares y 289 públicas. E1 56 por ciento de los programas de doctorado pertenecen al Padrón Nacional de Posgrado de Calidad (PNPC) 
del Consejo Nacional de Ciencia y Tecnología (Conacyt), a diferencia de las maestrías cuya pertenencia es de apenas 16 por ciento y donde se concentra el mayor número de programas brindados por instituciones particulares (Sánchez Soler, Herrera y Ponce, 2012).

- El crecimiento de los integrantes del Sistema Nacional de Investigadores (SNI) (el programa que incorpora a los investigadores en activo con doctorado en México, que laboran en universidades o centros de investigación) de 12,096 en 2006 a 23,316 en 2015, además de que incluye a 700 miembros que radican en el exterior, no compensa en absoluto el éxodo de emigrantes altamente calificados del país, toda vez que en 2012 el monto de repatriados fue de apenas 93 (Sánchez Soler, Herrera y Ponce, 2012).

- En 2010, a México se le otorgaron 14,576 patentes, de las cuales 93.5 por ciento correspondieron a solicitantes extranjeros (Villa Rivera, 2012). En similar tenor el país se ubica entre los países más dependientes de tecnología foránea del mundo (véase gráfica 5).

\section{GRÁFICA 5}

Relación de autosuficiencia en patentes por país, 2013

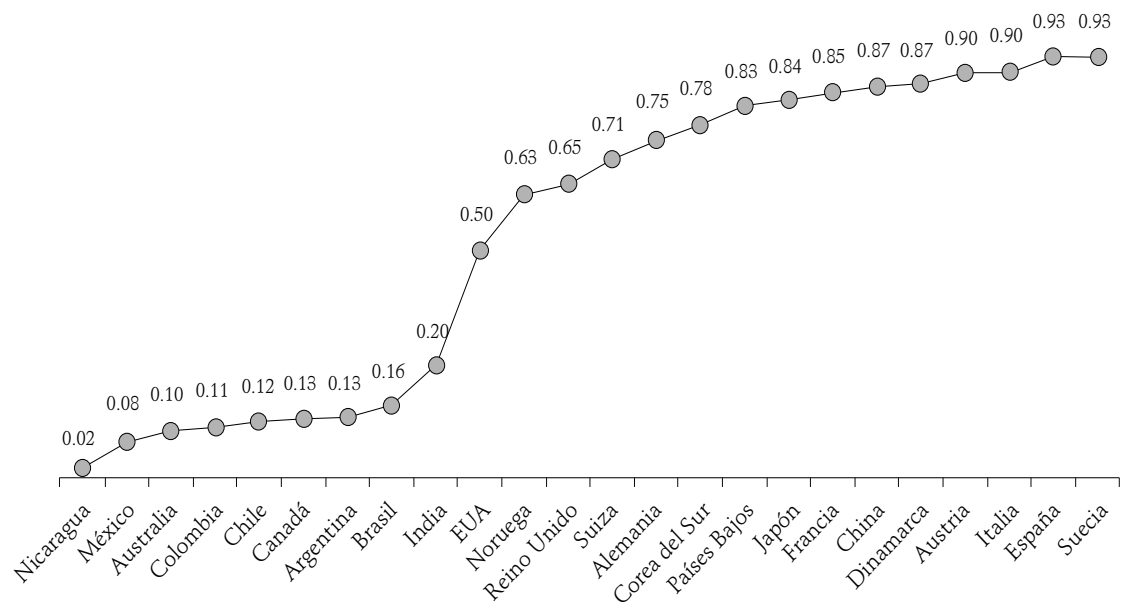

Nota: La tasa de autosuficiencia se mide como el cociente del total de patentes solicitadas por residentes entre el total de patentes solicitadas.

Fuente: SIMDE, UAz. Elaborado con base en WIPO Patent Report: Statistics on Worldwide Patent Activity, The World Bank, http://data.worldbank.org/indicator/IP.PAT.RESD/countries?display=default 
- En contraste con este último saldo, que en sí mismo alude a la prácticamente nula existencia de un Sistema Nacional de Innovación, es pertinente señalar que México se ubica en el primer sitio de América Latina en lo referente a corredores migratorios de inventores (Miguélez y Carsten, 2013). Asimismo, es oportuno consignar que una parte significativa de la actividad innovadora emprendida por científicos y tecnólogos mexicanos se realiza allende las fronteras, como lo demuestra el hecho de que una de cada tres patentes de connacionales se genera por residentes en el extranjero, donde, como se desprende de la gráfica 6, el país se sitúa en segundo lugar apenas por debajo de India en el plano internacional.

\section{GRÁFICA 6}

Patentes registradas por la diáspora, 2007-2012* (porcentajes)

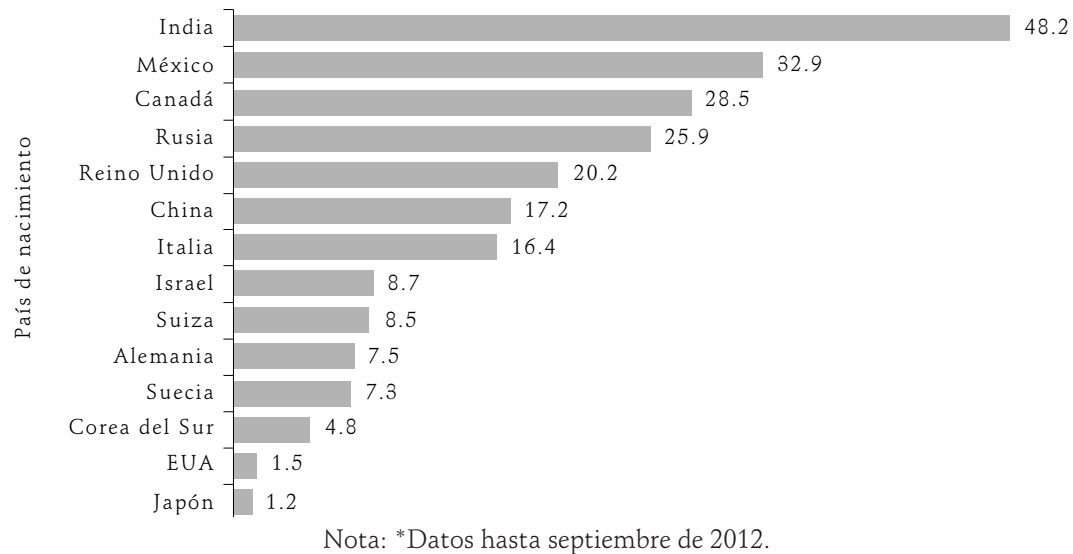

Fuente: Elaborado con datos de WIPO, «Migrant brainpower», The Economist, 27 de junio de 2015, en http://www.economist.com/news/international/21656175-migrant-brainpower? $\mathrm{frsc}=\mathrm{dg} \% 7 \mathrm{Cd}$

Queda claro, por tanto, que en el caso mexicano no sólo no se cumplen los supuestos de la llamada circulación de talentos, sino que el enorme caudal de inversión en educación superior, y particularmente en posgrado, que se ha venido realizando a partir de la década de 1990, aunque limitada en comparación con países de mayor desarrollo, ha resultado un tanto superflua (Gandini y Lozano, 2012). En efecto, sin considerar los déficits en calidad 
- sobre todo en el nivel de maestría donde, como vimos, se dio un boom en la oferta proveniente de instituciones privadas-, lo cierto es que el crecimiento de posgraduados mexicanos no tuvo correspondencia con transformaciones en el mercado laboral, en virtud de las limitaciones estructurales derivadas del modelo exportador de fuerza de trabajo barata prevaleciente en el país. En este contexto, los vínculos — de por sí limitados- entre el desarrollo científico-tecnológico y la economía doméstica tienden a desarticularse y desdibujarse aún más, restringiendo sobremanera la demanda nacional de fuerza de trabajo altamente calificada. Esta situación tiende a traducirse en una sobreoferta de profesionistas y personal científico-tecnológico, dando lugar a un significativo desperdicio de talentos y un creciente incremento de la emigración altamente calificada a Estados Unidos y otras latitudes. Esto resulta particularmente significativo en el caso de las áreas del conocimiento más directamente relacionadas con la innovación: ciencias, tecnologías, ingenierías y matemáticas. Si se considera, además, que los ingresos salariales y las oportunidades laborales para este segmento de la población - aunque relativamente menores que para los nativos y otros núcleos de inmigrantes - tienden a ser mejores en Estados Unidos (y otros países) que en México, es previsible que el éxodo del talento mexicano continúe su curso ascendente.

A lo anterior se agrega la elevada proporción de migrantes mexicanos que realizaron sus estudios de posgrado en Estados Unidos (aproximadamente 50 por ciento) y que en su mayoría fueron financiados con fondos públicos o privados de México. Este segmento poblacional, al igual que aquel formado en México y que reside en el extranjero, no encuentra condiciones propicias, tanto salariales como de desarrollo profesional, para retornar o vincularse desde el exterior en iniciativas y proyectos que contribuyan al desarrollo nacional. No resulta extraño, en este sentido, que apenas 5.6 por ciento de la masa total de retornados entre 2005 y 2010 haya correspondido a migrantes de alta calificación (Tuirán y Ávila, 2013b). 


\section{Conclusiones: la emergencia de unA NUEVA MODAlidAD DE DEPENDENCIA Y SUS DESAFÍOS}

La reestructuración de los sistemas de innovación en el marco de la globalización neoliberal constituye un mirador privilegiado para analizar y comprender el significado e implicaciones de la migración altamente calificada tanto para los países de origen y destino, como para los principales sujetos involucrados: corporaciones multinacionales, universidades, centros de investigación y los propios migrantes. Desde este mirador se evidencia la inoperancia y superficialidad de las nociones de fuga de cerebros, circulación de cerebros y ganancia de cerebros como categorías analíticas supuestamente explicativas del fenómeno.

Retomando la noción de exportación de fuerza de trabajo empleada para caracterizar al modelo exportador que se instaura en México, queda claro que la dinámica de reestructuración de los sistemas de innovación antes referida se inscribe en esta dinámica. De hecho, se configura como una etapa avanzada o superior de la misma, que opera en una doble vertiente: la exportación directa, vía migración de fuerza de trabajo altamente calificada y la exportación indirecta, mediante el establecimiento de maquiladoras científicas. Esta última vertiente es aún relativamente incipiente en el caso de México. Al respecto, Gallengher y Zarsky (2007), en un estudio sólidamente fundamentado y bastante revelador, ponen de relieve que la inversión extranjera directa en tecnologías de la información realizada en el llamado Silicon Valley mexicano ${ }^{3}$ no generó un foco de innovación capaz de generar impactos multiplicadores sobre la economía mexicana y operó, más bien, bajo la modalidad de una economía de enclave.

En un sentido más profundo, es pertinente advertir que la exportación de fuerza de trabajo encierra una nueva modalidad de intercambio desigual en el horizonte Norte-Sur (y en nuestro caso, entre México y Estados Unidos),

${ }^{3}$ Para algunos autores la región de Guadalajara constituye el Silicon Valley mexicano al contar con la presencia de 12 fabricantes de equipos originales, 16 proveedores de servicios de fabricación electrónica, decenas de centros de diseño y más de 700 compañías que fabrican electrónica (Manterola, 2008). 
cuya comprensión resulta crucial para develar los procesos de transferencia de excedentes implicados en el entramado estratégico/estructural que se teje en torno a las cadenas globales de mercancías y que están en la base de la reinserción asimétrica y subordinada de la economía mexicana a la estadounidense. No obstante la centralidad que el concepto de intercambio desigual tuvo en décadas pasadas para explicar la dinámica del desarrollo desigual y desentrañar la naturaleza del vínculo entre países desarrollados y periféricos o emergentes - tanto en el pensamiento de la Comisión Económica para América Latina (CEPAL) como entre los teóricos de la dependencia-, su inclusión en los análisis del capitalismo contemporáneo permanece como una asignatura pendiente. Es importante tener presente que el grueso de la discusión sobre intercambio desigual estuvo y permanece circunscrita al análisis de la división internacional del trabajo que confiere a la periferia el papel de proveedora de materias primas y a los países desarrollados el de suministradores de productos industrializados. Y si bien esta división continúa vigente para un número significativo de países periféricos, ha dejado de ser privativa de las relaciones centro-periferia. Algunos países periféricos de reciente industrialización — principalmente de la región asiática - fungen, cada vez más, como proveedores de bienes industrializados. Más importante aún resulta el hecho de que, a esta modalidad clásica de intercambio desigual, se ha agregado una nueva que bajo la égida de la globalización neoliberal cobra progresiva centralidad: la exportación, directa e indirecta, de fuerza de trabajo.

Para incursionar en el análisis de esta modalidad, en su doble vertiente, es preciso advertir que involucra mecanismos de intercambio desigual mucho más desfavorables para la periferia que aquellos implicados en el intercambio de materias primas por bienes manufacturados. Por un lado, la exportación indirecta de fuerza de trabajo asociada a la participación de las naciones periféricas en calidad de apéndices de las cadenas globales de mercancías conlleva una transferencia neta de las ganancias al exterior. Se trata de una forma extrema de intercambio desigual, ya que implica trasladar al exterior prácticamente la totalidad del excedente económico generado por la fuerza de trabajo empleada en las maquiladoras o plantas de ensamble. 
Mediante este mecanismo, que reedita la lógica del enclave exportador, se inhibe cualquier perspectiva de crecimiento económico y desarrollo derivada de las operaciones de exportación que, bajo el disfraz de exportaciones manufactureras, realiza la nación periférica. De hecho, su contribución efectiva al proceso de acumulación nacional se limita a una exigua derrama salarial -aprovechando los enormes diferenciales salariales entre países, en nuestro caso entre México y Estados Unidos-y, en el mejor de los casos, a un reducido impacto multiplicador por la vía del consumo. Más aún, la instalación y operación de plantas de ensamble en países periféricos viene acompañada de subsidios y exenciones tributarias con cargo al fondo de capital social del país en cuestión, así como de daños colaterales tales como el estrechamiento, diferenciación y precarización de los mercados laborales y la degradación ambiental. Otro aspecto de la exportación indirecta de fuerza de trabajo, que ha comenzado a cobrar fuerza en el contexto de los países periféricos o emergentes, es la creación de complejos científico/tecnológicos vinculados, como vimos, a la reestructuración de los sistemas de innovación en algunos de los países de mayor desarrollo, con Estados Unidos a la cabeza. A través de estos complejos, que funcionan bajo esquemas de subcontratación, asociación u otras formas de partnership, se transfieren al exterior bienes intangibles, que por desarrollarse bajo modalidades colectivas: share economy, commons economy y crowdsourcing economy, adquieren el carácter de «bienes comunes disfrazados» (Ramis, 2014: 112), los cuales tienen un valor y un significado estratégico aún mayor que las ganancias netas provenientes de la maquila y las plantas de ensamble. Nos referimos a la transferencia de capacidad de desarrollo y progreso, lo cual se materializa en ventajas competitivas y capacidades para generar ganancias extraordinarias.

La exportación directa de fuerza trabajo, vía migración laboral, implica la transferencia de los beneficios futuros anticipados que surgen de los gastos formativos y de reproducción social de la fuerza de trabajo que emigra. Estos costos — como se ha demostrado para el caso de México- no son compensados por el flujo de remesas (Delgado Wise, Márquez y Rodríguez, 2009). En términos demográficos, esta transferencia significa — para países periféricos que se ubican en un estadio avanzado de la transición 
demográfica - la exportación de su bono demográfico, es decir, la población en edad productiva que sirve de soporte para el sostenimiento de la población infantil y los adultos mayores. En un sentido más profundo, esta transferencia implica la pérdida del más importante recurso para la acumulación de capital en el país de origen: su fuerza laboral. Más todavía, la exportación de fuerza de trabajo altamente calificada exacerba el problema al reducir potencialmente las capacidades del país de origen para innovar en su propio beneficio e impulsar proyectos de desarrollo intensivos en tecnología, con pertinencia social y en armonía con el medio ambiente.

Analizar estas nuevas modalidades de intercambio desigual plantea un desafío teórico, metodológico y empírico, ya que requiere de cambios en la percepción y caracterización de las categorías utilizadas convencionalmente para interpretar el capitalismo contemporáneo. Con todo, sin desconocer los significativos aportes de la CEPAL para avanzar en la comprensión de este fenómeno (sobre todo en lo concerniente al papel central otorgado al progreso técnico), es importante traer a colación que las teorías marxistas del intercambio desigual, en su doble vertiente - en sentido estricto y amplio- proveen un sólido y fértil piso categorial para avanzar en la conceptualización de las modalidades emergentes de intercambio desigual implicadas en la exportación de fuerza de trabajo. Por un lado, el intercambio desigual en sentido estricto pone en el centro del análisis los diferenciales salariales derivados de los obstáculos a la movilidad poblacional (que, en términos más precisos, se refieren a los diferenciales en las tasas de plusvalor) y, por el otro, el intercambio desigual en sentido amplio agrega a estos diferenciales aquellos emanados de las diversas composiciones orgánicas del capital, i.e. los diferenciales en progreso científico/tecnológico, de los países involucrados. Tómese en consideración que la internacionalización del capital en el marco de la globalización neoliberal busca incesantemente abaratar los costos laborales -incluyendo aquellos relacionados con la fuerza de trabajo altamente calificada-y maximizar las transferencias de excedentes entre países periféricos y desarrollados, a partir, precisamente, de aprovechar y profundizar los diferenciales salariales.

$26 \frac{\text { SEGUNDO SEMESTRE } 2015}{\text { MIGRACIÓN Y DESARROLLO NÚM. } 25}$ 
Todo esto nos lleva a concluir que la exportación de fuerza de trabajo altamente calificada proveniente de países periféricos o emergentes, lejos de constituir una opción en la que todos ganan - como lo sugiere la noción de circulación de talentos en boga - configura una nueva modalidad de dependencia, particularmente expoliadora y depredadora. Ante este escenario, el gran desafío para los países periféricos consiste en contrarrestar las dinámicas que desvinculan a la diáspora calificada de los procesos de desarrollo nacional para construir un proyecto alternativo capaz de confrontar el orden sistémico imperante en beneficio de las clases trabajadoras y de los sectores populares en general.

Y en esta última perspectiva, resulta fundamental tener presente que tanto en la teoría como en la práctica comienzan a vislumbrarse posibilidades fácticas de emancipación y reorientación del intelecto colectivo hacia rutas alternativas de transformación social radical. Al respecto, Alberto Bialakowsky y un destacado grupo de colaboradores nos plantean que

La cooperación intelectual colectiva se torna una fuente de valorización que puede ser reapropiada a través de una praxis científica co-participativa, que incorpore la práctica dialógica como herramienta de transformación, promoviendo metodologías que orienten a las fuerzas productivas en la exploración de cogniciones colectivas emancipadoras (Bialakowsky, 2013: 11).

Resistir la tendencia en curso hacia la privatización y apropiación corporativa de los bienes comunes intangibles es una tarea urgente e insoslayable que implica, entre otras cosas: a) promover una currícula y agendas de investigación alternativas mediante redes del conocimiento interrelacionadas entre sí y en alianza con organizaciones y movimientos sociales, y b) desarrollar formas novedosas de creación y diálogo de saberes y vinculación de éstos con el tejido productivo y social bajo un prisma anticapitalista, antiimperialista y anticolonial (Santos, 2009; Acosta, 2008; Hall, 2011; Mendonça Ferreira, 2012). 


\section{Bibliografía}

Acosta, Alberto (2008), «El buen vivir, una oportunidad por construir», en Ecuador Debate. Innovaciones y retos constitucionales, Quito, CAAP, pp. 33-47.

Battelle (2012), 2013 Global R\&D Funding Forecast, Columbus, Battelle.

Bauwens, Michael (2005), "The Political Economy of Peer Production», Post-Autistic Economics Review, núm. 37, pp. 33-44.

Bialacowsky, Alberto (director) (2013), Coproducción e intelecto colectivo. Investigando para el cambio con la fábrica, el barrio y la universidad, Buenos Aires, Editorial Teseo.

Boletín Informativo Secab (mayo-junio, 2014), «Boletín informativo mayo-junio 2014», Convenio Andrés Bello, disponible desde junio 5, 2014 en http://convenioandresbello.org/inicio/boletines/mayo_junio_2014-ascyt_ecuador/

Burgesmani, Robert, Christensen, Clayton M. y Steven C. Wheelwright (2009), Strategic Management of Technology and Innovation (5th edition), Boston, McGraw-Hill.

Casillas, Rodolfo (2012), «La mundialización del delito. Redes de tráfico y trata de personas en México», Nueva Sociedad, núm. 241, pp. 122-132.

Castillo, Manuel A. (2005), "Fronteras, migración y seguridad en México», Alteridades, núm. 15, pp. 51-60.

Chesbrough, Henry (2008), «Open Innovation: A New Paradigm for Understanding Industrial Innovation», en Henry Chesbrough, Win Vanhaverbeke y Joel West (eds.), Open Innovation: Researching a New Paradigm, Oxford, Oxford University Press.

Cypher, James y Raúl Delgado Wise (2011), Mexico's Economic Dilemma: The Developmental Failure of Neoliberalism, Rowman \& Littlefield Publishers.

Cyranoski, Gilbert; Heidi Ledford, Anjali Nayar y Mohammed Yahia (2011), «The $\mathrm{PhD}$ Factory. The World is Producing More PhDs than Ever Before. Is it Time to Stop?», Nature, vol. 472, 21 de abril.

Delgado Wise, Raúl (2003), «La educación superior en México bajo la égida neoliberal: elementos para un diagnóstico» en Daniel Cazés y Raúl Delgado Wise (coords.), Hacia una politica de Estado para la educación superior en México, México, Miguel Ángel Porrúa, pp. 11-21.

(2013), «The Migration and Labor Question Today: Imperialism, Unequal Development and Forced Migration», Monthly Review, vol. 65, núm. 2, pp. 25-38.

$28 \frac{\text { SEGUNDO SEMESTRE } 2015}{\text { MIGRACIÓN Y DESARROLLO NÚM. } 25}$ 
Delgado Wise, Raúl, Humberto Márquez y Héctor Rodríguez (2009), «Seis tesis para desmitificar el nexo entre migración y desarrollo», Migración y Desarrollo, vol. 6, núm. 12, pp. 27-52.

Delgado Wise, Raúl y James Cypher (2007), «The Strategic Role of Mexican Labor Under NAFTA: Critical Perspectives on Current Economic Integration», The Annals of the American Academy of Political and Social Science, núm. 615, pp. 120-142.

Delgado Wise, Raúl y Humberto Márquez (2007), «The Reshaping of Mexican Labor Exports under NAFTA: Paradoxes and Challenges», International Migration Review, vol. 41, núm. 3, pp. 656-679.

Dumont, Jean-Christophe, Gilles Spielvogel y Sarah Widmaier (2010), International Migrants in Developed, Emerging and Developing Countries. An Extended Profile, París, OECD.

Foladori, Guillermo (2014), "Ciencia ficticia», Estudios Críticos del Desarrollo, vol. IV, núm. 7, pp. 41-66.

Foster, John B., Robert W. Macchesney y Jamil Jonna (2011), «The Global Reserve Army of Labor and the New Imperialism,» Monthly Review, vol. 63, núm. 6, pp. $1-15$.

Freeman, Richard B. (2008), "The New Global Labor Market», Focus, vol. 26, núm. 1, pp. 1-6.

Galama, Titus y James Hosek (2008), U.S. Competitiveness in Science and Technology, Santa Mónica, Rand Corporation.

Gallengher, Kevin y Lyuba Zarsky (2007), The Enclave Economy: Foreign Investment and Sustainable Development in Mexico's Silicon Valley, Cambridge, Massachusetts, Mit Pess.

GANDINI, Luciana y Fernando Lozano (2012), «La migración mexicana calificada en perspectiva comparada: el caso de los profesionistas con posgrado en Estados Unidos, 2001-2010» en Telésforo Ramírez y Manuel Ángel Castillo (eds.), México ante los recientes desafíos de la migración internacional, México, Conapo, pp. 87-126.

Hall, Budd L. (2011), «Towards a Knowledge Democracy Movement: Contemporary Trends in Community-University Research Partnerships», Rizoma Freireano, núm. 9, pp. 1-18.

Ibarra Colado, Eduardo y Luis Porter (2007), «Costos de la evaluación en escenarios de continuidad: lecciones mexicanas sobre las disputas con los mercaderes del templo del saber», Espacio Abierto, vol. 16, núm. 1, pp. 61-88. 
IbARRA Colado, Eduardo (2005), "Origen de la empresarialización de la universidad: el pasado de la gestión de los negocios en el presente del manejo de la universidad», Revista de la Educación Superior, vol. XXXIV (2), núm. 134, pp. 13-37.

Instituto Nacional de Estadística, Geografía e Informática (INEGI) (2015), Encuesta Nacional de Ocupación y Empleo (ENOE), "Consulta interactiva de indicadores estratégicos (InfoLaboral), 2005 a 2014 tercer trimestre», en http://www.inegi. gob.mx

Manterola, Manuel Antonio (2008), "Jalisco: El milagroso «Silicon Valley» de México», Revista Electroindustria, en http://www.emb.cl/electroindustria/ articulo. $m v c^{2} \times$ xid $=878 \&$ tip $=7$

Márouez, Humberto y Raúl Delgado Wise (2011), «Una perspectiva del sur sobre capital global, migración forzada y desarrollo alternativo», Migración y Desarrollo, vol. 9, núm. 16, pp. 3-42.

Mendonça Ferreira, Sebastiao Darlan (2012), «Evolution and Future of the Knowledge Commons: Emerging Opportunities and Challenges for Less Developed Societies», IKM Working Paper, núm. 15, pp. 1-35.

MeYer, Jean-Baptiste (2011), "La sociología de las diásporas del conocimiento», en Lucas Luchilo (eds.), Más allá de la fuga de cerebros: Movilidad, migración y diásporas de argentinos calificados, Buenos Aires, Universidad de Buenos Aires, pp. 91-114.

Miguélez, Ernest y Fink Carsten (2013), «Measuring the International Mobility of Inventors: A New Database», Working Paper, núm. 8, World Intellectual Property Organization.

National Science Board (2012), Science and Engineering Indicators Digest, Arlington, The National Science Board.

Organisation for Economic Co-operation and Development (OECD) (2008), Open Innovation in Global Networks, Copenhaguen, OECD.

Parthasarathi, Ashok (2002), "Tackling the Brain Drain from India's Information and Communication Technology Sector: the Need for a New Industrial, and Science and Technology Strategy», Science and Public Policy, vol. 29, núm. 2, pp. 129-136.

Partnership for a New American Economy (2012), Patent Pending. How Immigrants are Reinventing the American Economy, a report from the partnership for a new American economy, en http://www.renewoureconomy.org/patent-pending

$30 \frac{\text { SEGUNDO SEMESTRE } 2015}{\text { MIGRACIÓN Y DESARROLLO NÚM. } 25}$ 
PAssel, Jeffrey y Cohn D’Vera (2011), «Unauthorized Immigrant Population: National and State Trends, 2010», Pew Hispanic Center, Pew Research Center, en http://www.pewhispanic.org/files/reports/133.pdf

Ramis, Álvaro (2014), «Los bienes comunes intangibles en el capitalismo cognitivo», Recerca. Revista de Pensament i Análisi, España, núm. 15, pp. 109-129.

Rand Corporation (2008), U.S. Competitiveness in Science and Technology, Santa Mónica, C.A, Rand Corporation.

Rodrícuez, Roberto (2009), «Migración de personal altamente calificado de México a Estados Unidos: una exploración del fenómeno», Revista Electrónica de Investigación Educativa, vol. 11, núm. 2, pp. 1-34.

SÁnchez Soler, María Dolores, Jorge Herrera y Luis Ponce (2012), «II Simposio de becarios y ex becarios del Conacyt», Edición Europa, Parlamento Europeo Estrasburgo, presentación en power point, PNPC, Conacyt, noviembre.

SAntos, de Sousa Boaventura (2009), Epistemología del Sur, México, Siglo XXI Editores. SaXenian, Anne Lee (1996), Regional Advantage: Culture and Competition in Silicon Valley and Route 128, Boston: Harvard University Press. (2002), Local and Global Networks of Immigrant Professsionals in Silicon Valley, San Francisco, Public Policy Institute of California. (2006), The New Argonauts: Regional Advantage in a Global Economy, Boston, Harvard University Press.

Sturgeon, Timothy J. (2003), "What Really goes on in Silicon Valley? Spatial Clustering and Dispersal in Modular Production Networks», Journal of Economic Geography, vol. 3, núm. 2, pp. 199-225.

Tello, Carlos (1986), «La economía mexicana: hacia el tercer milenio», Nexos, núm. 226, pp. 47-55.

TUIRÁN Rodolfo y José Luis Ávila (2013a), «Migración calificada México-Estados Unidos: desafíos y opciones de política», ponencia presentada en la mesa redonda «Hacia una agenda de investigación sobre migración y desarrollo para el futuro de México», en el marco del proyecto Hacia Dónde va la Ciencia en México, Ccc, AMC, Conacyt, Zacatecas, 10 de abril.

(2013b), «La migración calificada entre México-Estados Unidos. Desafíos y opciones de política», Migración y Desarrollo, vol. 11, núm. 22 (en prensa).

U.S. Department of Homeland Security (2014), 2013 Yearbook of Immigration Statistics, Washington, D.C., U.S. Department of Homeland Security, Office of Immigration Statistics. 
United States Patent and Trademark Office (2012), «Patenting by Organizations», en http://www.uspto.gov/web/offices/ac/ido/oeip/taf/topo_12.pdf

Villa Rivera, José Enrique (2012), «Estado actual de la ciencia, la tecnología y la innovación en México», presentación en power point, Conacyt, en http:// academiadeingenieriademexico.mx/archivos/v_congreso/conferencias/dr_jose enrique_villa_rivera-ciencia_y_tecnoloia.pdf 